Pacific Journal of Mathematics

cANONICAL EXTENSIONS OF MEASURES AND THE
EXTENSION OF REGULARLY OF CONDITIONAL

Luis Harvey blake 


\title{
CANONICAL EXTENSIONS OF MEASURES AND THE EXTENSION OF REGULARITY OF CONDITIONAL PROBABILITIES*
}

\author{
LouIs H. Blake
}

\begin{abstract}
Let $(\Omega, \mathfrak{A}, P)$ be a probability space with $\mathscr{B}$ a sub $\sigma$-field of $\mathfrak{A}$. Let $\mathfrak{A}^{\prime} \equiv \sigma(\mathfrak{A}, H)$, the $\sigma$-field generated by $\mathfrak{A}$ and $H$, where $H$ is a subset of $\Omega$ not in $\mathfrak{U} . \quad P_{e}$ will be called a simple extension of $P$ to $\mathfrak{X}^{\prime}$ if $P_{e}$ is a probability measure on $\mathfrak{A}^{\prime}$ which agrees with $P$ on $\mathfrak{A}$.

The purpose of this paper is to use a particular type of simple extension called a canonical extension, denoted as $P_{c}$ to examine under what conditions the regularity of the conditional probability $P^{\mathfrak{B}}$ will extend to the regularity of $P_{c}^{\mathfrak{B}}$. Also, if $\mathfrak{A}$ is countably generated and $P_{c}^{\mathfrak{B}}$ is regular, a characterization of $P_{c}^{\mathfrak{B}}$ in terms of $P^{\mathfrak{B}}$ will be given.
\end{abstract}

The terminology in the following definitions will be used throughout this paper.

Definition. The conditional probability of a set $A \in \mathfrak{A}$ given the $\sigma$-field $\mathfrak{B}$ is a $\mathfrak{B}$-measurable function denoted by $P^{\mathfrak{B}}(\cdot, A)$ such that for every $B \in \mathfrak{B}$

$$
\int_{B} P^{\Re}(\cdot, A) d P_{\mathfrak{B}}=P(A B) .
$$

DEFINITION. The conditional probability (given $\mathfrak{B}$ ) is the collection of functions

$$
\left\{P^{\mathfrak{B}}(\cdot, A) \mid A \in \mathfrak{N}\right\} \text {. }
$$

This collection is denoted by $P^{\Re}$.

Definition. For $A \in \mathfrak{A}$, a version of $P^{\mathfrak{g}}(\cdot, A)$ is a selection from the equivalence class of $P^{\mathscr{Q}}(\cdot, A)$ which will be denoted by $p(\cdot, A \mid \mathfrak{B})$.

Definition. A version of the conditional probability $P^{\Re}$ is a function $p(\cdot, \cdot \mid \mathfrak{B})$ on $X \times \mathfrak{A}$ such that for each $A \in \mathfrak{X} p(\cdot, A \mid \mathfrak{B})$ is a version of $P^{\mathfrak{B}}(\cdot, A)$. Also $p(w, \cdot \mid \mathfrak{B})$ will denote a section of $p(\cdot, \cdot \mid \mathfrak{B})$ at $w \in X$.

Definition. A conditional probability $P^{\mathfrak{B}}$ is called regular if there exists a version, $p(\cdot, \cdot \mid \mathfrak{B})$, such that $p(w, \cdot \mid \mathfrak{B})$ is a measure on $\mathfrak{V} P_{\mathfrak{B}}$ a.e.

Before the main body of the paper is presented, it should be 
observed that the regularity of $P^{\mathfrak{B}}$ itself is not in general sufficient to insure the regularity of $P_{c}^{\mathfrak{s}}$; for example, see [2], p. 210.

Finally, the scope of this paper is limited to results on canonical extensions. A forthcoming paper will deal with the preservation of regularity for simple extensions.

The main results. Observe that the $\sigma$-field

$$
\mathfrak{Y}^{\prime}=\left\{A_{1} H+A_{2} H^{\mathfrak{c}} \mid A_{1}, A_{2} \in \mathfrak{X}\right\},
$$

and make

Definition 1. Let $A^{\prime}$ be any element of $\mathfrak{A}^{\prime}$ with $A^{\prime}=A_{1} H+A_{2} H^{c}$ for some $A_{1}$ and $A_{2}$ in $\mathfrak{A}$. A simple extension will be called a canonical extension, $P_{c}$, if there exists a number $\alpha$ between zero and one with $\beta=1-\alpha$ and $K \in \mathfrak{A}$ so that

$$
\begin{aligned}
& \text { (a) } A^{\prime} K^{c} \in \mathfrak{A} \\
& \text { (b) } P_{c}\left(A^{\prime}\right)=P\left(A^{\prime} K^{c}\right)+\alpha P\left(A_{1} K\right)+\beta P\left(A_{2} K\right)
\end{aligned}
$$

with $P_{c}$ a well defined probability measure on ' $\mathfrak{A}$ '.

Marczewski and Los have shown, [4], that for any subset of $X$ not in $\mathfrak{A}$, say $H$, there always exists a canonical extension $P_{c}$ on $\mathfrak{A} \mathfrak{X}^{\prime}$. (It has been shown by the author in [1] that there exist many simple extensions which are not canonical.)

REMARK 2. One way of obtaining the set $K$ of Definition 1 is by letting $K_{1}$ be an element of $\mathfrak{A}$ such that $\left(P K_{1}\right)=P_{*}(H)$ and $K_{2}$ be an element of $\mathfrak{A}$ such that $P\left(K_{2}\right)=P^{*}(H)$ with $K_{1} \subset H \subset K_{2}$. Then, simply define $K=K_{2} \backslash K_{1}$. (See [2], P. 71). Observe that there exists another $K^{\prime} \in \mathfrak{A}$ which will extend $P$ canonically to $\mathfrak{A}^{\prime}$ as in Definition 1 if and only if $P\left(K \Delta K^{\prime}\right)=0$.

Lemma 3. Let $(X, \mathfrak{A}, P), \mathfrak{B} \subset \mathfrak{A}$ and $\mathfrak{A}^{\prime}=\sigma(\mathfrak{A}, H)$ be given. Let $p(\cdot, \cdot \mid \mathfrak{B})$ be a version of $P^{\mathscr{B}}$ which makes $P^{\mathfrak{B}}$ regular. Let $P_{c}$ be a canonical extension of $P$ to $\mathfrak{A}^{\prime}$ with $\alpha, \beta$ and $K$ as in Definition 1. Suppose for $w, P_{\mathfrak{B}}$ a.e., $p_{c}(w, \cdot \mid \mathfrak{B})$ is a canonical extension of $p(w, \cdot \mid \mathfrak{B})$ to $\mathfrak{2}^{\prime}$ with the same $\alpha$ and $\beta$ and $K$ as $P_{c}$. Then, $P_{c}^{\mathfrak{B}}$ is regular.

Proof. It will suffice to produce a version of $P_{c}^{\mathfrak{B}}$ which makes $P_{c}^{\Re}$ regular.

Let $A^{\prime} \in \mathfrak{Q}^{\prime}$ with $A^{\prime}=A_{1} H+A_{2} H^{c}$ for some $A_{1}$ and $A_{2}$ in $\mathfrak{A}$. For $w, P_{\mathfrak{B}}$ a.e.,

$$
\begin{aligned}
P_{c}\left(w, A^{\prime} \mid \mathfrak{B}\right)= & p\left(w, A^{\prime} K^{c} \mid \mathfrak{B}\right)+\alpha p\left(w, A_{1} K \mid \mathfrak{B}\right) \\
& +\beta p\left(w, A_{2} K \mid \mathfrak{B}\right) .
\end{aligned}
$$


Thus it is immediate from (3.1) that $p_{c}\left(\cdot, A^{\prime} \mid \mathfrak{B}\right)$ is a $\mathfrak{B}$-measurable function for all $A^{\prime} \in \mathfrak{Q}^{\prime}$ and for $w, P_{\mathfrak{g}}$ a.e., $p_{c}(w, \cdot \mid \mathfrak{B})$ is a measure on $\mathfrak{H}^{\prime}$. It is also clear that for $A^{\prime} \in \mathfrak{A}^{\prime}$ and $B \in \mathfrak{B}$

$$
\int_{B} P_{c}\left(\cdot, A^{\prime} \mid \mathfrak{B}\right) d P_{c}=P_{c}\left(A^{\prime} B\right) \text {. }
$$

For, integrating the right side of (3.1) with respect to $P$ gives

$$
P\left(A^{\prime} K^{c} B\right)+\alpha P\left(A_{1} K B\right)+\beta P\left(A_{2} K B\right)=P_{c}\left(A^{\prime} B\right) .
$$

But $P_{c}=P$ on $\mathfrak{B}$ and so the integral of the right side of (3.1) is exactly the left side of (3.2) .

Hence, $p_{c}(\cdot, \cdot \mid \mathfrak{B})$ is the desired version.

Theorem 4. Let $(X, \mathfrak{A}, P), \mathfrak{B}$, and $\mathfrak{H}$ be as in Lemma 3. Suppose $P^{\mathfrak{B}}$ is regular and $p(\cdot, \cdot \mid \mathfrak{B})$ is a version such that

$$
p(w, \cdot \mid \mathfrak{B}) \text { is a measure } P_{\mathfrak{g}} \text { a.e. }
$$

(4.2) $p(w, \cdot \mid \mathfrak{B}) \ll Q\left(P_{\mathfrak{8}}\right.$ a.e.) where $Q$ is a probability measure on $\mathfrak{A}$.

Let $P_{c}$ be a canonical extension of $P$ to $\mathfrak{X}^{\prime}$ with respect to $\alpha, \beta$ and $K$ as in (1.1). Then, $P_{c}^{g}$ is regular.

Proof. Suppose $K^{\prime}=K_{2} \backslash K_{1}$, where $K_{1} \subset H \subset K_{2}, Q_{*}(H)=Q\left(K_{1}\right)$ and $Q^{*}(H)=Q\left(K_{2}\right)$. Consider any set $A \subset K_{2} \backslash H$ where $A \in \mathfrak{A}$. $Q(A)=0$. By (4.2) $p(w, A \mid \mathfrak{B})=0\left(P_{\mathfrak{g}}\right.$ a.e.) and so therefore $P(A)=0$ also. Similarly, if $B \subset H \backslash K_{1}$, where $B \in \mathfrak{A}$, then $Q(B)=0$ and hence $p(w, B \mid \mathfrak{B})=0$ and so $P(B)=0$ also. Thus $p^{*}(w, H \mid \mathfrak{B})=p\left(w, K_{2} \mid \mathfrak{B}\right)\left(P^{\mathfrak{B}}\right.$ a.e. $)$ and $p\left(w, K_{1} \mid \mathfrak{B}\right)=p_{*}(w, H \mid \mathfrak{B})\left(P_{\mathfrak{B}}\right.$ a.e. $)$. Also, $P\left(K_{1}\right)=P_{*}(H)$ and $P^{*}(H)=P\left(K_{2}\right)$. According to Remark $2, p(w, \cdot \mid \mathfrak{B})$ can be extended canonically to $\mathfrak{A}^{\prime}$ with respect to $\alpha, \beta$ and $K^{\prime}$ and by Lemma 3 the proof is complete.

The following result is a consequence of Theorem 4 .

Theorem 5. Let $(X, \mathfrak{A}, P), \mathfrak{B}$ and $\mathfrak{A}^{\prime}$ be as in Lemma 3. Suppose $P^{\mathfrak{Y}}$ is regular and $p(\cdot, \cdot \mid \mathfrak{B})$ is a version such that

(5.1) $p(w, \cdot \mid \mathfrak{B})$ is a measue $P_{\mathfrak{s}}$ a.e.

(5.2) there exists a sequence $\left\{w_{n}\right\}_{n=1}$ such that for every $\varepsilon>0$ and any $w\left(P_{\mathfrak{B}}\right.$ a.e. $)$ there is an $w_{n}$ with

$$
\sup _{A \in \mathscr{X}}\left|p(w, A \mid \mathfrak{B})-p\left(w_{n}, A \mid \mathfrak{B}\right)\right|<\varepsilon .
$$

Let $P_{c}$ be a canonical extension of $P$ to $\mathfrak{A}^{\prime}$ with $\alpha, \beta$ and $K$ as in (1.1). Then, $P_{c}^{:}$is regular. 
Proof. Let $Q$ be a probability measure defined as

$$
\sum_{n=1}^{\infty} \frac{1}{2^{n}} p\left(w_{n}, \cdot \mid \mathfrak{B}\right) \text {. }
$$

Condition (5.2) insures that $p(w, \cdot \mid \mathfrak{B}) \ll Q P_{\mathfrak{g}}$ a.e. and the result follows from Theorem 4.

The following proposition is presented for the sake of completeness.

Let $(X, \mathfrak{A}, P)$ be a probability space with $(X, \overline{\mathfrak{A}}, \bar{P})$ denoting the completion. Suppose $H$ is in $\overline{\mathfrak{A}}$ but not in $\mathfrak{A}$. Let $\mathfrak{A}^{\prime}=\sigma(\mathfrak{A}, H)$.

Proposition 6. Let $(X, \mathfrak{A}, P), \mathfrak{B} \subset \mathfrak{A}$, and $\mathfrak{A}{ }^{\prime}=\sigma(\mathfrak{A}, H)$ with $H \in$ $\overline{\mathfrak{A}} \backslash \mathfrak{A}$ be given. Let $P_{1}$ denote the restriction of $\bar{P}$ to $\mathfrak{A}^{\prime}$. If $P^{\mathfrak{B}}$ is regular then so is $P_{1}^{\mathfrak{B}}$.

The proof can be viewed as an easy consequence of Lemma 3 and is therefore omitted.

The remainder of this paper is devoted to the single

TheOREM 7. Let $(X, \mathfrak{A}, P)$ be a probability space with $\mathfrak{A}$ generated by a countable field, $\mathscr{A}$. Let $\mathscr{A}^{\prime}$ be the field generated by $\mathscr{A}$ and $H$ and $\mathfrak{X}^{\prime}=\sigma\left(\mathscr{A}^{\prime}\right)$. Let $P_{c}$ be a canonical extension of $P$ to $\mathfrak{X}^{\prime}$ with respect to $\alpha, \beta$ and $K$ and suppose $P_{c}^{\mathfrak{g}}$ is regular where $\mathfrak{B} \subset \mathfrak{A}$. Then, there exists a version $p^{\prime}(\cdot, \cdot \mid \mathfrak{B})$ of $P_{c}^{\mathfrak{g}}$ such that $P_{\mathfrak{g}}$ a.e. $p^{\prime}(w, \cdot \mid \mathfrak{B})$ is a probability measure which is a canonical extension of $p^{\prime}(w, \cdot \mid \mathfrak{B}) \mid \mathfrak{A}$ with respect to the same $\alpha, \beta$ and $K$ that are associated with $P_{c}$.

The following lemmas are introduced before presenting the main body of the proof.

Lemma 8. Let $(X, \mathfrak{A}, P)$ be a probability space with $\mathfrak{Y} \mathfrak{X}^{\prime}=\sigma(\mathfrak{H}, H)$ and $P_{e}$ an arbitrary simple extension of $P$ to $\mathfrak{X}^{\prime}$. Let $K$ be the set associated with a canonical extension of $P$ to $\mathfrak{P}^{\prime}$ as in Remark 2 . Then, for each set $A \in \mathfrak{P}$ there exist constants $\alpha_{A}$ and $\beta_{A}$ with $0 \leqq \alpha_{A} \leqq 1$ and $0 \leqq \beta_{A} \leqq 1$ and such that $P_{e}(A H K)=\alpha_{A} P(A K)$ and $P_{e}\left(A H^{c} K\right)=\beta_{A} P(A K)$.

Proof. For $A \in \mathfrak{A}, A K \supset A H K$. If $P(A K) \neq 0$, then $\alpha_{A}=P_{e}(A H K) / P(A K)$; otherwise, let $\alpha_{A}$ be arbitrary between zero and one. $\beta_{A}$ is obtained similarly.

Lemma 9. Assume the hypothesis of Lemma 8. Let $\mathscr{A}$ be a field which generates $\mathfrak{A}$ and $\mathscr{A}^{\prime}$ the field generated by $\mathscr{A}$ and $H$. Let $\alpha(\mathscr{A}) \equiv \sup _{A \in \mathscr{A}} \alpha_{A}$ and $\beta(\mathscr{A}) \equiv \sup _{A \in \mathscr{A}} \beta_{A}$. Then, a necessary and sufficient condition that $P_{e}$ be a canonical extension of $P$ to $\mathfrak{P}^{\prime}$ is that 
$\alpha(\mathscr{A})=\alpha_{X}$ or $\beta(\mathscr{A})=\beta_{X}$ for some $\mathscr{A}$ which generates $\mathfrak{A}$.

Proof. Necessity is obvious and only sufficiency is proved. Let $\mathscr{A}$ be some field which generates $\mathfrak{A}$ and $\alpha(\mathscr{A})=\alpha_{X}$. (For simplicity, write $\alpha(\mathscr{A})=\alpha$.) By hypothesis,

$$
P_{e}(H K)=\alpha P(K) \text {. }
$$

For $A \in \mathscr{A}$ it follows by Lemma 8 that

$$
P_{e}(A H K)=\alpha_{A} P(A K)
$$

and

$$
P_{e}\left(A^{c} H K\right)=\alpha_{A^{c}} P\left(A^{c} K\right) .
$$

The following equalities also hold

$$
\begin{gathered}
\alpha P(K)=\alpha P(A K)+\alpha P\left(A^{c} K\right) \\
P_{e}(H K)=P_{e}(A H K)+P_{e}\left(A^{c} H K\right) .
\end{gathered}
$$

By (9.1) - (9.4) it follows that

$$
0=\left(\alpha-\alpha_{A}\right) P(A K)+\left(\alpha-\alpha_{A^{c}}\right) P\left(A^{c} K\right) .
$$

If $P(A K)=0$, set $\alpha_{A}=\alpha$ or if $P\left(A^{c} K\right)=0$, set $\alpha_{A^{c}}=\alpha$ (see Lemma 8). Otherwise, (9.5) forces $\alpha-\alpha_{A}=\alpha-\alpha_{A^{c}}=0$ and hence for any $A \in$ $\mathscr{A}, P_{e}(A H K)=\alpha P(A K)$.

Next, the fact that $P_{e}\left(A H^{c} K\right)=\beta P(A K), \beta=1-\alpha$, is immediate from the following chain of equalities:

$$
\begin{aligned}
& P(A)=P_{e}\left(A H+A H^{c}\right)=P_{e}\left(\left(A H+A H^{c}\right) K^{c}\right)+P_{e}(A H K) \\
& \left.\quad+P_{e} A H^{c} K\right)=P\left(A K^{c}\right)+\alpha P(A K)+P_{e}\left(A H^{c} K\right) .
\end{aligned}
$$

Hence, where $\mathscr{A}^{\prime}=\left\{A_{1} H+A_{2} H^{c} \mid A_{i} \in \mathscr{A} i=1,2\right\}, A^{\prime}$ in $\mathscr{A}^{\prime}$ can be written as $A^{\prime}=A_{1} H+A_{2} H^{c}$ and it follows that

$$
P_{e}\left(A^{\prime}\right)=P\left(A^{\prime} K^{c}\right)+\alpha P\left(A_{1} K\right)+\beta P\left(A_{2} K\right) .
$$

Finally, let

$$
\begin{aligned}
& \dot{\phi}_{\alpha}=\left\{A \in \mathfrak{A} \mid P_{e}(A H K)=\alpha P(A K)\right\} \\
& \phi_{\beta}=\left\{A \in \mathfrak{N} \mid P_{e}\left(A H^{c} K\right)=\beta P(A K)\right\} .
\end{aligned}
$$

Both $\phi_{\alpha}$ and $\phi_{\beta}$ are monotone classes containing $\mathscr{A}$; hence, the proof is complete by the monotone class theorem (see [3], p. 60).

Theorem 7 can now be proved.

Proof. For $w \in X, P_{\mathfrak{B}}$ a.e., and $A \in \mathscr{A}$, write 


$$
p^{\prime}(w, A H K \mid \mathfrak{B})=\alpha_{w, A} p(w, A K \mid \mathfrak{B})
$$

where $0 \leqq \alpha_{w, A} \leqq 1$ as in Lemma 8 and $p(w, \cdot \mid \mathfrak{B})$ will be written for $\left.p^{\prime}(w, \cdot \mid \mathfrak{B})\right|_{\mathscr{\Re}}$. For fixed $A \in \mathscr{A}, \alpha_{w, A}$ is a $\mathfrak{B}$-measurable function where

$$
\begin{aligned}
& \alpha_{w, A}=p^{\prime}(w, A H K \mid \mathfrak{B}) / p(w, A K \mid \mathfrak{B}) \text { for } p(w, A K \mid \mathfrak{B}) \neq 0 \\
& \alpha_{w, A}=\alpha \text { if } p(w, A K \mid \mathfrak{B})=0 .
\end{aligned}
$$

(In (7.1) $\alpha$ is associated with $P_{c}$ and by Lemma $9, \alpha=\sup _{A \in \Re} \alpha_{A}$ ).

For $A \in \mathscr{A}$ let

$$
U_{A} \equiv\left\{w \mid \alpha_{w, A}>\alpha\right\}
$$

Observe that $U_{A}$ is contained in the complement of the set of $w$ 's where $p(w, A K \mid \mathfrak{B})=0$.

Also, $U_{A} \in \mathfrak{B}$ (see (7.1)). Hence, since $P_{c}$ is a canonical extension, it follows that

$$
\alpha P\left(A U_{A} K\right)=P_{c}\left(A U_{A} H K\right)=\int_{U_{A}} p^{\prime}(w, A H K \mid \mathfrak{B}) d P_{c} .
$$

Also,

$$
\begin{aligned}
& \int_{U_{A}} p^{\prime}(w, A H K \mid \mathfrak{B}) d P_{c}=\int_{U_{A}} \alpha_{w, A} p(w, A K \mid \mathfrak{B}) d P \geqq \\
& \int_{U_{A}} \alpha p(w, A K \mid \mathfrak{B}) d P=\alpha P\left(A U_{A} K\right) .
\end{aligned}
$$

Hence, the defining properties of $U_{A}$ together with (7.3) and (7.4) say that $P\left(U_{A}\right)=0$.

If $L_{A} \equiv\left\{w \mid \alpha_{w, A}<\alpha\right\}$, then an argument similar to the preceding one shows $P\left(L_{A}\right)=0$.

Hence, for each set $A \in \mathscr{A}$, there exists a $P_{\mathfrak{s}}$ null set on the complement of which $\alpha_{w, A}=\alpha$. But where $\mathscr{A}$ is countable, it follows that there exists a $P_{\mathfrak{r}}$ null set, $N$, on the complement of which $\alpha_{w, A}=\alpha$ for all $A \in \mathscr{A}$. Thus,

$$
p^{\prime}(w, A H K \mid \mathfrak{B})=\alpha p(w, A K \mid \mathfrak{B})
$$

for all $w \in N^{c}$ and $A \in \mathscr{A}$.

Finally, if $\alpha_{w} \equiv \sup _{A \in \mathscr{A}} \alpha_{w, A}$, then it is immediate from (7.5) that $P_{\mathfrak{B}}$ a.e. $\alpha_{w}=\alpha=\alpha_{X}$ and by Lemma 9 the theorem is proved.

\section{REFERENCES}

1. L. H. Blake, The Preservation of Regularity of Conditional Probabilities, Doctoral Dissertation, University of Maryland, 1969.

2. P. R. Halmos, Measure Theory, Eleventh Printing, D. Van Nostrand Company, Inc., Princeton, New Jersey, 1966.

3. M. Loeve, Probability Theory, Third Edition, D. Van Nostrand Company, Inc., Pri- 
nceton, New Jersey, 1963.

4. E. Marczewski and J. Loś, 'Extensions of measure'' Fund. Math., 36, pp. 267-276.

Received November 30,1970. This paper is based in part on the author's doctoral dissertation completed at the University of Maryland under the direction of Professor R.E.'Syski.

Worcester Polytechnic Institute 



\section{PACIFIC JOURNAL OF MATHEMATICS}

\section{EDITORS}

H. SAMELSON

Stanford University

Stanford, California 94305

C. R. Hовву

University of Washington

Seattle, Washington 98105
J. DugundJI

Department of Mathematics

University of Southern California

Los Angeles, California 90007

RICHARD ARENS

University of California

Los Angeles, California 90024

\section{ASSOCIATE EDITORS}
E. F. BECKENBACH
B. H. NeumanN
F. WOLF
K. YOSHIDA

\section{SUPPORTING INSTITUTIONS}

UNIVERSITY OF BRITISH COLUMBIA

CALIFORNIA INSTITUTE OF TECHNOLOGY

UNIVERSITY OF CALIFORNIA

MONTANA STATE UNIVERSITY

UNIVERSITY OF NEVADA

NEW MEXICO STATE UNIVERSITY

OREGON STATE UNIVERSITY

UNIVERSITY OF OREGON

OSAKA UNIVERSITY
UNIVERSITY OF SOUTHERN CALIFORNIA

STANFORD UNIVERSITY

UNIVERSITY OF TOKYO

UNIVERSITY OF UTAH

WASHINGTON STATE UNIVERSITY

UNIVERSITY OF WASHINGTON

$\stackrel{*}{*} \stackrel{*}{*} \stackrel{*}{*}{ }^{*}$ AMERICAN MATHEMATICAL SOCIETY

NAVAL WEAPONS CENTER 


\section{Pacific Journal of Mathematics}

\section{Vol. 41, No. $1 \quad$ November, 1972}

Anatole Beck and Peter Warren, Weak orthogonality.................

Jonnie Bee Bednar and Howard E. Lacey, Concerning Banach spaces whose duals are abstract $L$-spaces.............................

Louis Harvey Blake, Canonical extensions of measures and the extension of regularity of conditional probabilities..........................

R. A. Brooks, Conditional expectations associated with stochastic processes ..........................................

Theodore Allen Burton and Ronald Calvin Grimmer, On the asymptotic behavior of solutions of $x^{\prime \prime}+a(t) f(x)=e(t) \ldots \ldots \ldots \ldots \ldots \ldots$

Stephen LaVern Campbell, Operator-valued inner functions analytic on the closed disc ............................................

Yuen-Kwok Chan, A constructive study of measure theory...

Alexander Munro Davie and Bernt Karsten Oksendal, Peak interpolation sets for some algebras of analytic functions ...................

H. P. Dikshit, Absolute total-effective $\left(N, p_{n}\right)(c, 1)$ method ...............

Robert E. Edwards, Edwin Hewitt and Kenneth Allen Ross, Lacunarity for

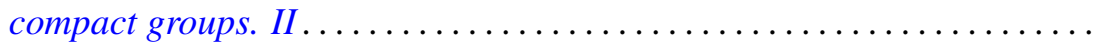

James Daniel Halpern, On a question of Tarski and a maximal theorem of Kurepa

Gerald L. Itzkowitz, A characterization of a class of uniform spaces that admit an invariant integral

Mo Tak Kiang, Semigroups with diminishing orbital diameters ..

Glenn Richard Luecke, A class of operators on Hilbert space ...

R. James Milgram, Group representations and the Adams spectral sequence. . .

G. S. Monk, On the endomorphism ring of an abelian p-group, and of a large subgroup...

Yasutoshi Nomura, Homology of a group extension ...

R. Michael Range, Approximation to bounded holomorphic functions on strictly pseudoconvex domains...

Norman R. Reilly, Inverse semigroups of partial transformations and $\theta$-classes.

Chris Rorres, Strong concentration of the spectra of self-adjoint operators

Saharon Shelah, A combinatorial problem; stability and order for models and theories in infinitary languages.

George Gustave Weill, Vector space decompositions and the abstract

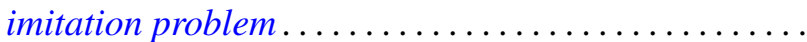

